\title{
Applications of the Hansen Solubility Parameter for Cellulose
}

\author{
Jinyan Lang, ${ }^{\mathrm{a}} \mathrm{Na}$ Wang, ${ }^{\mathrm{a}}$ Xinhui Wang, ${ }^{\mathrm{a}}$ Yili Wang, ${ }^{\mathrm{a}}$ Guorong Chen, ${ }^{\mathrm{b}}$ and \\ Heng Zhang a,b*
}

\begin{abstract}
Based on the solubility parameter theory, the Hansen solubility parameters of various solvents were calculated and compared to predict the solubility of cellulose in various solvents, which illustrates the feasibility of Hansen solubility parameters to predict the solubility of cellulose in solvents. This paper aims to make a more accurate prediction in advance when finding suitable cellulose solvent system, and then to reduce the burden of cellulose solvent selection.
\end{abstract}

Keywords: Cellulose; Dissolution; Solubility parameter; Deep Eutectic Solvents

Contact information: a: College of Marine Science and Biological Engineering, Qingdao University of Science \& Technology, Qingdao Shandong 266042, China; b: Key Laboratory of Fire Retardant Materials of Fujian Province, Xiamen University, Xiamen 361005, China;

*Corresponding author: hgzhang@ sina.com

\section{INTRODUCTION}

Natural cellulose is a very promising macromolecular compound, which can be used as a biomass resource for basic chemical raw materials. Cellulose has a highly crystalline structure; therefore, cellulose must be dissolved in a specific solvent in practical applications and then catalytically converted into a specific product before application. The structure of hydrogen bonds between and within the cellulose molecules is stable, which makes the cellulose difficult to dissolve. Relying on experimental trial and error to find a suitable cellulose solvent is time-consuming and laborious. It follows that screening the ideal solvent for a specific substance through solubility parameters may be a more efficient screening method.

Many researchers have verified the feasibility of solubility parameter theory. Ramos et al. (2013) effectively measured the solubility parameters of oils and used the calculated solubility parameters to predict the compatibility of oils. Hatefi et al. (2018) established a general model based on Hansen solubility parameters to predict the solubility of paracetamol in co-dissolution systems at different temperatures. Zhao et al. used reversed-phase chromatography and the Hansen solubility sphere method to determine the solubility parameters of ionic liquids. They found that the latter provides higher solubility parameter values. Zhang et al. (2019) effectively screened out suitable solvents for lowtemperature degradation of lignocellulosic biomass based on the Hansen solubility parameter theory. Therefore, the rational use of solubility parameters can help to find and screen the most efficient cellulose solvent to a certain extent.

Cellulose solvents have been developed for a long time, with various types, and new cellulosic solvents are constantly being developed. At present, there is still a lack of research on the solubility parameters of cellulose by solvents. In this paper, the solubility parameter theory was used as the theoretical basis, and the solubility parameters were used 
to compare the solubility of natural cellulose by different solvents. Solubility parameters were calculated to explore the feasibility of selecting cellulose solvents and to conduct a comparative study of the solubility parameters of cellulose in a new green solvent-deep eutectic solvents (DES) system.

\section{Theoretical Basis of Solubility Parameters}

Hildebrand proposed the concept of solubility parameter $(\delta)$, which was originally defined by the square root of the cohesive energy density of the liquid. The parameter can be used as a physical constant to predict the relative compatibility of liquid compounds (Hildebrand and Scott 1950).

At present, the solubility parameters of common solvents can be checked in the relevant references to predict the compatibility of polymer materials with a certain solvent (You and Shi 2001). The closer the solubility parameter of the compound, the better the mutual solubility effect. However, the presence of a hydrogen bond structure in the solvent will increase the critical difference, which means that even if the solubility parameter difference between the solvent and the material is large, the solvent still can dissolve the material. Therefore, if the solubility parameters are used reasonably and the solubility parameters of cellulose have been determined, only the solubility parameters of the candidate cellulose solvents need to be determined to screen cellulose solvents more quickly and efficiently.

The solubility parameter proposed by Hildebrand can be regarded as being the sum of all the interaction forces between the molecules, but this results in the calculated solubility parameters of the two substances being similar, but the actual dissolution effect is not ideal. The interaction force between molecules is not single; rather, it is the sum of the dispersion force, the polar force, and the hydrogen bond adhesion force. If the goal is to dissolve a certain solute in a solvent smoothly, it is necessary not only that the sum of the molecular forces be similar, but each component of the forces between the two molecules also needs to be distinguished and individually measured, which is more in line with the principle of "similar and compatible".

As originally proposed in 1966, the Hansen solubility parameter system is based on three component forces: the dispersion force, the polar force, and the hydrogen bond adhesion force (Hansen 1967). The Hansen solubility parameter formula is as follows,

$$
\delta^{2}=\delta_{d}^{2}+\delta_{p}^{2}+\delta_{h}^{2}
$$

where $\delta$ is the solubility parameter of the substance, $\delta_{d}$ is the partial parameter of the dispersion force, $\delta_{p}$ is the partial parameter of the polar force, and $\delta_{h}$ is the partial parameter of the hydrogen bond adhesion. The units in each case are $\left(\mathrm{J} / \mathrm{cm}^{3}\right)^{1 / 2}$.

So far, the Hansen solubility parameters of most substances have been calculated through calculations and experiments. One can directly refer to the Hansen solubility parameter table for use. The group contribution method (Carlos 2018) can be used to calculate the parameter components of the specific substance not in the table after knowing the basic physical data of the substance. The values of $\delta_{d}, \delta_{p}$, and $\delta_{h}$, can be calculated by the following formulas,

$$
\delta_{d}=\frac{\sum_{i} G_{d i}}{V} \quad \delta_{p}=\frac{\sqrt{\sum_{i} G_{p i}^{2}}}{V} \quad \delta_{h}=\sqrt{\frac{\sum_{i} E_{h i}}{V}}
$$


where $V$ is molar volume $\left(\mathrm{cm}^{3} / \mathrm{mol}\right)$ of solvent substance molecules, $G_{\mathrm{d}}$ is the dispersion force component $\left(\mathrm{J} / \mathrm{cm}^{3}\right)^{1 / 2}, G_{\mathrm{p}}$ is the polar force component $\left(\mathrm{J} / \mathrm{cm}^{3}\right)^{1 / 2}$, and $E_{\mathrm{h}}$ is the hydrogen bond adhesive force component $\left(\mathrm{J} / \mathrm{cm}^{3}\right)^{1 / 2}$.

In much of the recent research and experiments, the solvent used to dissolve a substance is not a single solvent system in most cases, but a mixed solvent system formed by a combination of two or more different solvents. If two different solvents are mixed, then there is no need to make a new measurement of the solubility of the mixed solvent. After obtaining the parameters of each solvent, the solubility parameters of the mixture can be calculated according to its respective known parameter values and mixing ratio, and the actual solubility of the mixed solvent can be predicted. This approach can be used so as to minimize the errors and losses that may occur in the case of trial and error.

The solubility parameter of the mixed solvent system cannot be directly checked in the Hansen solubility parameter table, so it needs to be further calculated. The calculation formula is as follows.

$$
\begin{aligned}
& \delta_{s}=\sum_{i=1}^{n} \phi_{i, s} \delta_{i, s} \\
& \sum_{i=1}^{n} \phi_{i, s}=1
\end{aligned}
$$

In these equations, $\varphi_{i, s}$ is molar mass fraction of component $\mathrm{i}, \delta_{i, s}$ is the solubility parameter of component $\mathrm{i},\left(\mathrm{J} / \mathrm{cm}^{3}\right)^{1 / 2}$.

\section{CALCULATION OF SOLUBILITY PARAMETERS}

\section{Selection of Cellulose Solvents}

The following three groups of representative solvents were selected in this study.

The first group was the known cellulose solvents: lithium chloride/N,Ndimethylacetamide $(\mathrm{LiCl} / \mathrm{DMAc})=1: 1$, sodium hydroxide/urea/aqueous solution=1:1, $\mathrm{H}_{2} \mathrm{O} / \mathrm{N}$-methylmorpholine- $\mathrm{N}$-oxide $\left(\mathrm{H}_{2} \mathrm{O} / \mathrm{NMMO}\right)=1: 1$, ionic liquid [BMIM]Cl;

The second group was commonly used solvents: deionized water $\left(\mathrm{H}_{2} \mathrm{O}\right)$, ethyl ether $\left(\mathrm{Et}_{2} \mathrm{O}\right)$, ethanol (ethanol), carbon tetrachloride $\left(\mathrm{CCl}_{4}\right)$;

The third group was deep eutectic solvents: urea/choline chloride (U-DES) $=1: 1$, oxalic acid/choline chloride $(\mathrm{O}-\mathrm{DES})=1: 1, \mathrm{p}$-toluenesulfonic acid/choline chloride (T$\mathrm{DES})=1: 1$, and malonic acid/choline chloride $(\mathrm{M}-\mathrm{DES})=1: 1$.

\section{Calculation of Solubility Parameters}

In this study, the Hansen solubility parameters were calculated to compare the values of the parameters to analyze the solubility of cellulose in the solvent, and the standard data of each substance at $25^{\circ} \mathrm{C}$ were selected for calculation.

Table 1. The Combined Parameters of Cellulose Hansen Solubility

\begin{tabular}{|l|c|c|c|c|}
\hline & $\delta_{\mathrm{d}} /\left(\mathrm{J} / \mathrm{cm}^{3}\right)^{1 / 2}$ & $\delta_{\mathrm{p}} /\left(\mathrm{J} / \mathrm{cm}^{3}\right)^{1 / 2}$ & $\delta_{\mathrm{h}} /\left(\mathrm{J} / \mathrm{cm}^{3}\right)^{1 / 2}$ & $\delta /\left(\mathrm{J} / \mathrm{cm}^{3}\right)^{1 / 2}$ \\
\hline Microcrystalline cellulose & 8.27 & 8.00 & 13.88 & 18.03 \\
\hline
\end{tabular}


Hansen solubility cannot deal with the problem of different crystal faces of cellulose. It is regarded as only a group of properties such as dispersion force, hydrogen bonding force, and polar force. The solubility parameters of cellulose were measured by Lee through Inverse Gas Chromatography, as shown in Table 1 (Lee et al. 1991).

The exact parameters of the corresponding solvent can be obtained both experimentally and theoretically. The solubility parameters measured by experiment are more accurate, but according to its basic physical properties, more accurate theoretical values can be obtained by calculation. The purpose of this study is to obtain the theoretical values through calculation and to calculate the theoretical solubility parameters of cellulose and each solvent by group contribution method.

\section{RESULTS AND DISCUSSION}

\section{Comparison of Hansen Solubility Parameters}

According to Hansen's solubility parameter theory, cellulose can be dissolved in a solvent, and the parameters of the cellulose and the solvent need to be similar. That is to say, the two need not only be similar in solubility parameter values, but also in their dispersion power parameter values, polar force parameter values, and hydrogen bond adhesive force parameter values. Table 2 lists the solubility parameter values of cellulose and those of selected solvents. Among them, the last column is the absolute difference between the solubility of cellulose and cellulose solvent.

Table 2. Combination Parameter Table of Solubility of Each Substance

\begin{tabular}{|c|c|c|c|c|c|}
\hline Solvents & $\delta_{\mathrm{d}} /\left(\mathrm{J} / \mathrm{cm}^{3}\right)^{1 / 2}$ & $\delta_{\mathrm{p}} /\left(\mathrm{J} / \mathrm{cm}^{3}\right)^{1 / 2}$ & $\delta_{\mathrm{h}} /\left(\mathrm{J} / \mathrm{cm}^{3}\right)^{1 / 2}$ & $\delta /\left(\mathrm{J} / \mathrm{cm}^{3}\right)^{1 / 2}$ & $\begin{array}{c}\text { Absolute } \\
\text { difference }\end{array}$ \\
\hline cellulose & 8.27 & 8.00 & 13.88 & 18.03 & 0 \\
\hline $\mathrm{DMAc}$ & 7.85 & 11.94 & 8.68 & 16.72 & 1.31 \\
\hline $\mathrm{NMMO}$ & 8.60 & 8.64 & 15.37 & 19.62 & 1.59 \\
\hline$[\mathrm{BMIM}] \mathrm{Cl}$ & 9.27 & 6.26 & 7.32 & 13.58 & 4.45 \\
\hline carbamide & 12.66 & 17.11 & 15.20 & 26.15 & 8.12 \\
\hline $\mathrm{H}_{2} \mathrm{O}$ & 15.50 & 16.00 & 42.30 & 47.80 & 29.77 \\
\hline $\mathrm{Et}_{2} \mathrm{O}$ & 7.10 & 1.40 & 2.50 & 7.60 & 10.43 \\
\hline $\mathrm{ethanol}_{\mathrm{CCl}}$ & 15.80 & 8.80 & 19.40 & 26.50 & 8.47 \\
\hline $\mathrm{O}-\mathrm{DES}$ & 17.80 & 0 & 0.60 & 17.80 & 0.95 \\
\hline $\mathrm{M}-\mathrm{DES}$ & 16.40 & 0.40 & 1.29 & 16.46 & 1.57 \\
\hline $\mathrm{T}-\mathrm{DES}$ & 19.83 & 0.44 & 1.48 & 19.89 & 1.86 \\
\hline $\mathrm{U}-\mathrm{DES}$ & 19.99 & 0.38 & 1.02 & 20.02 & 1.99 \\
\hline
\end{tabular}

The following is an analysis of each item of data, the first is the analysis and comparison of solubility parameter values.

As shown in Fig. 1, the best solubility parameter ring is drawn based on the solubility parameter value of cellulose. Under ideal conditions, the solubility parameter of the solvent on the optimal solubility parameter ring is equal to that of cellulose, so it is the most ideal to dissolve cellulose. And the farther the distance from this parameter ring is, the worse the dissolving ability of cellulose is predicted to be. The solvents dispersed on both sides of the optimal solubility parameter ring also have the ability to dissolve cellulose. The smaller the relative distance between the two sides of the optimal solubility parameter 
ring, the better the solubility. It can be seen from Fig. 1 that the solubility parameter values of DMAc, NMMO, ionic liquid [BMIM]Cl, urea, carbon tetrachloride, and O-DES, UDES, T-DES, and M-DES are all close to each other. Compared with cellulose, in theory, these solvents have the ability to dissolve cellulose.

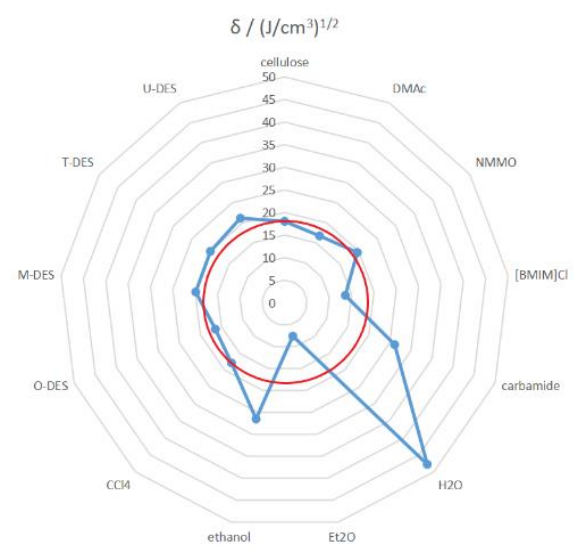

Fig. 1. Solubility parameters of cellulose and various solvents

It can be seen from Fig. 2 (a) that, in terms of the dispersion force components, the four solvents of DMAc, NMMO, ionic liquid [BMIM]Cl, and diethyl ether are predicted to be the best solvents. The dispersion force components parameters of the four solvents are close to that of cellulose. The four kinds of DES have larger dispersion force components parameters, so their solubility to cellulose is relatively poor.

As can be seen from Fig. 2 (b), there is a large gap between cellulose and all solvents in terms of polar force component parameters. The polar force component parameters of ethanol, NMMO, and ionic liquid [Bmim] $\mathrm{Cl}$ are the most similar to cellulose, so in theory, the three solvents are the optimal cellulose solvent in terms of polar force component. The polar force component parameters of four kinds of DES are different from those of cellulose.

As can be seen from Fig. 2 (c), due to the hydrogen bond structure of cellulose itself, its hydrogen bond adhesion component parameter value is relatively large. NMMO and urea are closest to cellulose in the hydrogen bond adhesion component, so it has the best solubility in those systems. The hydrogen bond adhesion component parameters of other solvents considered here are smaller than those of cellulose, and the parameters of water are the largest.

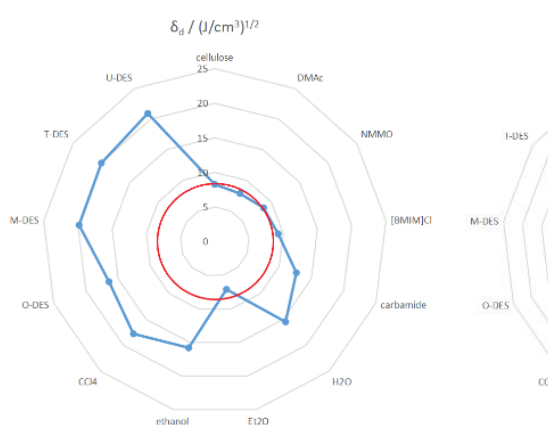

(a)

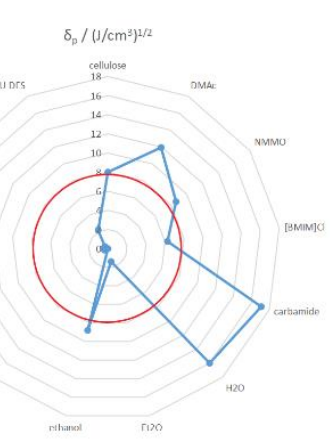

(b)

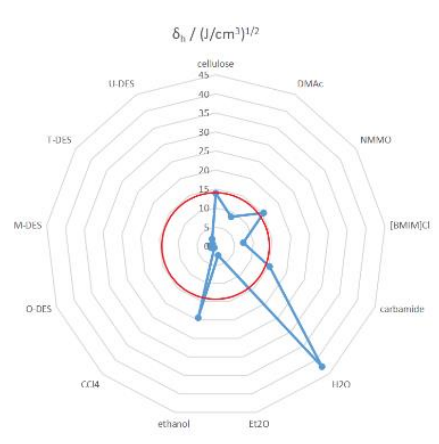

(c)

Fig. 2. Dispersion force component parameters (a), polar force component parameters (b) and hydrogen bond adhesion component parameters (c) of cellulose and various solvents 
By comparing the solubility parameters in the table with the three component parameters, although the solubility parameters of DMAc, NMMO, Ionic Liquid [BMIM]Cl, carbon tetrachloride, and four types of DES (O-DES, U-DES, T-DES and M-DES) are close to cellulose in terms of values, their actual solubility is greatly different. Carbon tetrachloride has no solubility to cellulose due to zero polar force component and close to zero hydrogen bond bonding force parameter. Although diethyl ether and ethyl alcohol are close to cellulose in some component parameters, their solubility parameters are different from cellulose, so they do not have the ability to dissolve cellulose. Water is different from cellulose in solubility parameters with respect to all three component parameters, so it has the least ability to dissolve cellulose. DMAc, NMMO, and Ionic Liquid [BMIM]Cl are very close to the data of cellulose in solubility parameters and three parameter components, so they also have the best cellulose solubility, which can be said to be the optimal solvents in theory at present. In addition, the theoretical value of NMMO is more favorable than the others. Although the solubility parameters of the four types of DES (O-DES, U-DES, TDES, and M-DES) are close to cellulose, there is a gap with cellulose in three component parameters. Therefore, the four types of DES have the ability to dissolve cellulose in theory, but the dissolution effect is poor, and it is not the best solvent for cellulose. In addition, for cellulose solubility, O-DES has the best effect, while U-DES has the worst. T-DES and MDES are somewhere in between.

\section{Parameter Score and Trapezoid Triangle Diagram}

In order to better explain the component factors of solubility parameters, Jean P. Teas designed a very famous equilateral triangle, namely the trapezoid diagram (Si 2012). He first assumed that the solubility parameter values of all liquids are the same, and the difference in solubility depends on the ratio of the constituent components rather than the size of the parameter. Therefore, the trapezoid triangle diagram expresses Hansen's combination parameters in the form of fractions, and the conversion formula is as follows.

The scores of various parameters of cellulose and solvent are calculated and displayed on the ladder triangle diagram, which can compare the solubility of the solvent to cellulose. The trapezoid triangle diagram constructed using the parameter scores can more intuitively reflect the solubility of cellulose in its solvent.

$$
f_{d}=\frac{\delta_{d}}{\delta_{d}+\delta_{p}+\delta_{h}} \quad f_{p}=\frac{\delta_{p}}{\delta_{d}+\delta_{p}+\delta_{h}} \quad f_{h}=\frac{\delta_{h}}{\delta_{d}+\delta_{p}+\delta_{h}}
$$

In the formula, $f_{d}$ is dispersive force parameter score, $f_{p}$ is polar force parameter score, $f_{h}$ is hydrogen bond adhesive force parameter score. The sum of the scores of the three parameters is constant at 100 .

$$
f_{d}+f_{p}+f_{h}=100
$$

According to the calculated parameters of the dispersion force, the polar force and the hydrogen bond adhesion force of the cellulose and the solvent, the following trapezoidal triangle diagram of the cellulose and the solvent is obtained.

The labels in Fig. 3 correspond to the positions of cellulose and each solvent in the trapezoid triangle. The circle in the figure indicates the selection range of the solubility parameter of cellulose solvent. In theory, all solvents within this range have the possibility of dissolving cellulose. According to the actual position of each solvent, it can be seen that 
the solubility parameters of N,N-dimethylacetamide, $\mathrm{N}$-methylmorpholine-N-oxide, ionic liquid $[\mathrm{BMIM}] \mathrm{Cl}$, and urea are all within this range. They are ideal cellulose solvents. Water, ether, ethanol, and carbon tetrachloride are not within this range, so they do not have the ability to dissolve cellulose. The four types of DES are also not within this range, but combined with the comparison of solubility, it shows that although DES has the ability to dissolve cellulose, it is not the best cellulose solvent. This is almost consistent with the itemized data analysis in the previous article.

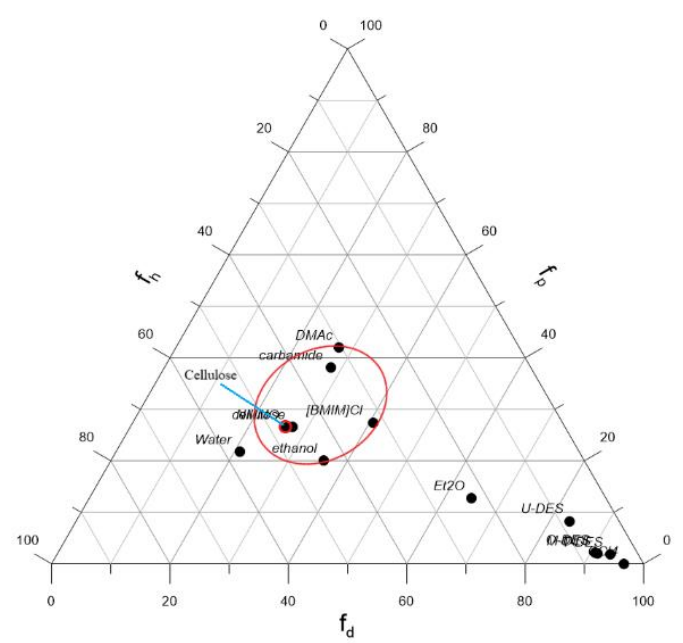

Fig. 3. Ladder triangle diagram of cellulose and solvent

In summary, the proportions of each component of cellulose in the solubility parameters are $0.2743,0.2653$, and 0.4604 , which indicates that the hydrogen bond bonding force is the dominant force, which is related to its special hydrogen bond network structure. The difference between the dispersion force and the polar force is small. Dispersion force is the force that the offset charge causes molecules to affect neighboring molecules. Polar force is the force that the strong polarity of some of the atoms inside the molecule makes the molecule attract or repel neighboring molecules. Polarity is relatively stable, because the polar molecules promote an organized arrangement. The dispersion force and the polar force are the forces formed by the cellulose molecules themselves and are related to their constituent elements and structure. However, the process of solvent dissolving solute is essentially that the solvent molecules overcoming the interaction force between the solute molecules, and the interaction force between the solvent molecules is also disintegrated by the solute molecules. In this way, the solvent molecules and the solute molecules can penetrate each other and disperse each other stably and evenly. At this time, the solute will be dissolved in the solvent smoothly. In the four solvent systems, the dispersion force component of DMAc and urea is close to that of cellulose, the polar force component of [BMIM] $\mathrm{Cl}$ is close to that of cellulose, and both components of NMMO are close to that of cellulose. Therefore, they can affect the intermolecular force of cellulose. Solvent molecules can penetrate the cellulose interior and weaken the interaction between macromolecules, allowing cellulose molecules to be distributed smoothly among the solvent molecules, forming a stable solution. At the same time, as compared with the dispersion force, polar force has a more stable feature, two kinds of force component were similar, while cellulose is more easily dissolved in polar solvent, the stability of force can better promote the cellulose molecules and solvent molecules dispersion and combination. 
From another perspective, the hydrogen bonding force can be seen as a special kind of polarity, it can also be seen that cellulose is more susceptible to polar forces and is more soluble in polar solvents. In addition, the four solvents can also break the hydrogen bond structure of cellulose, which also facilitates the binding of cellulose molecules and solvent molecules.

\section{The Actual Solubility of Cellulose in Some Solvents}

Finally, part of the references related to the research of cellulose dissolution is integrated. Qi et al. (2016) dissolved wheat straw material pretreated with ethanol-dilute sulfuric acid in the $\mathrm{LiCl} / \mathrm{DMAc}$ solvent system, and the dissolution extent reached $84.4 \%$. Li et al. (2012) obtained a higher saccharification rate $(96.5 \%)$ in the NMMO aqueous medium by ultrasonic-assisted treatment of cellulose after pretreatment in $15 \%$ and $20 \%$ (w/v) NMMO solutions. Ang et al. (2011) used ionic liquid [BMIM]Cl to dissolve rice husk cellulose, and the cellulose dissolution was 31.3\%. Han (2014) used ionic liquid [BMIM]Cl as a solvent under high temperature conditions to catalyze the conversion of microcrystalline cellulose into glucose and 5-HMF, and finally obtained a glucose yield of $31 \%$. The yield of 5-HMF even reached $61 \%$. Ai et al. (2020) used the deep eutectic solvent $\mathrm{ChCl} / \mathrm{Gly}$ to treat sorghum bagasse, and the high glucose and xylose yield of saccharified sorghum by enzymatic hydrolysis was higher than 85\%. Lang et al. (2020) prepared ODES to dissolve cellulose, and the yields of glucose and 5-HMF reached the maximum, which were $23.5 \%$ and $29.8 \%$, respectively. Ling et al. (2020) pretreated Moso bamboo with the prepared $\mathrm{LA} / \mathrm{ChCl}$ and achieved the highest glucose yield of $79.07 \%$. Chen et al. (2018) used $\mathrm{ChCl} / \mathrm{Gly}$ to divide willow branches into three parts under relatively mild conditions: cellulose-rich pulp, lignin, and xylose-rich liquid. The pulp showed a high dissolution rate, and the glucose yield was about $89 \%$. These actual results also show that the predictions made in the previous article are accurate. The theoretical solubility parameters of several cellulose solvents are compared with the actual solubility as follows.

Table 3. Comparison of the Solubility Combination Parameters and the Actual Solubility of Each Substance

\begin{tabular}{|c|c|c|c|}
\hline Solvents & $\delta /\left(\mathrm{J}^{\mathrm{cm}}\right)^{1 / 2}$ & Absolute difference & Actual solubility \\
\hline DMAc & 16.72 & 1.31 & $84.4 \%$ (Qi et al. 2016) \\
\hline NMMO & 19.62 & 1.59 & $96.5 \%$ (Li et al. 2012) \\
\hline [BMIM]Cl & 13.58 & 4.45 & $\begin{array}{c}31.3 \% \text { (Ang et al. 2011) } \\
31 \% \text { (Han 2014) }\end{array}$ \\
\hline O-DES & 16.46 & 1.57 & $23.5 \%$ (Lang et al. 2020) \\
\hline ChCl / Gly & 25.68 & 7.65 & $\begin{array}{c}85 \% \text { (Ai et al. 2020) } \\
89 \% \text { (Chen et al. 2018) }\end{array}$ \\
\hline LA / ChCl & 19.78 & 1.75 & $79.07 \%$ (Ling et al. 2020) \\
\hline
\end{tabular}

\section{CONCLUSIONS}

1. Under ideal conditions, the Hansen solubility parameter can predict the solubility properties of solvent relative to solid materials of interest, thereby reducing a lot of experimental work, and the predicted results can show the solubility property of the solvent. 
2. The parameter values and component values of N,N-dimethylacetamide (DMAc), Nmethylmorpholine-N-oxide (NMMO), and certain ionic liquids are close to those of cellulose. They have the best dissolving effect on cellulose. And the four types of DES have the dissolving ability to cellulose, but they are not the best solvents. Among them, 1:1 oxalic acid/choline chloride (O-DES) has greater solubility for cellulose, while urea/choline chloride (U-DES) has less solubility for cellulose. The solubility parameters of $\mathrm{H}_{2} \mathrm{O}$, ethanol, and ether are far from those of cellulose. So they have basically no ability to dissolve cellulose.

\section{ACKNOWLEDGMENTS}

The authors are grateful for the support of the Shandong Provincial Key Research and Development Program (SPKR\&DP) (Grant No. 2019GGX102029) and open fund of the Key Laboratory of Fire Retardant Materials of Fujian Province (Grant No. FH202101).

\section{REFERENCES CITED}

Ai, B., Li, W., Woomer. J., Li, M., and Shi, J. (2020). "Natural deep eutectic solvent mediated extrusion for continuous high-solid pretreatment of lignocellulosic biomass," Green Chemistry 22(19), 6372-6383. DOI: 10.1039/D0GC01560A

Ang, T. N., Li, W. Y., Lee, K. M., Ngoh, G. C., and Min, G. L. (2011). "Efficiency of Ionic Liquids in the Ionic Liquids in the Dissolution of Rice Husk," Bioresources 6(4), 4790-4800. DOI: 10.15376/biores.6.4.4790-4800

Chen, Z., Reznicek, W. D., and Wan, C. (2018). "Deep eutectic solvent pretreatment enabling full utilization of switchgrass," Bioresource Technology 263, 40-48. DOI: 10.1016/j.biortech.2018.04.058

Han, H. Y. (2014). The Preparation of 5-HMF from Microcrystalline Cellulose in Ionic Liquid and Kinetics Study, Master's Thesis, Qingdao University of Science and Technology, Qingdao, China.

Hansen, C. M. (1967). "The three-dimensional solubility parameter-key to paint component affinities: I. Solvents, plasticizers, polymers, and resins," Journal of Paint Technology 39(505), 104-117.

Hatefi, A., Jouyban, A., Mohammadian, E., Acree, W. E., and Rahimpour, E. (2018). "Prediction of paracetamol solubility in cosolvency systems at different temperatures," Journal of Molecular Liquids 273, 282-291.

DOI: $10.1016 /$ j.molliq.2018.10.031

Hildebrand, J. H., and Scott, R. L. (1950). The Solubility of Nonelectrolytes, Reinhold, New York, NY.

Lang, J., Lu, J., Lan, P., Wang, N., and Zhang, H. (2020). "Preparation of 5-HMF in a DES/ethyl N-butyrate two-phase system," Catalysts 10(6), 636. DOI: 10.3390/catal10060636

Lee, H., Hak, L., and Luner, P. (1991). "The solubility parameter of cellulose and alkylketene dimer (AKD) determined by inverse gas chromatography," Journal of Wood Chemistry \& Technology 11(2), 247-261. DOI: 10.1080/02773819108050273 
Li, Q., Ji, G. S., Tang, Y. B., Gu, X. D., Fei, J. J., and Jiang, H. Q. (2012). “Ultrasoundassisted compatible in situ hydrolysis of sugarcane bagasse in cellulase-aqueous-Nmethylmorpholine-N-oxide system for improved saccharification," Bioresource Technology 107(9), 251-257. DOI: 10.1016/j.biortech.2011.12.068

Ling, Z., Guo, Z., Huang. C., Yao, L., and Xu, F. (2020). "Deconstruction of oriented crystalline cellulose by novel levulinic acid based deep eutectic solvents pretreatment for improved enzymatic accessibility," Bioresource Technology 305, article no. 123025. DOI: 10.1016/j.biortech.2020.123025

Qi, G., Lian, X., Bo, W., Lin, X., and Chen, X. (2016). "Improvement and characterization in enzymatic hydrolysis of regenerated wheat straw dissolved by LiCl/DMAc solvent system," Applied Biochemistry and Biotechnology 181(1), 1-15. DOI: $10.1007 / \mathrm{s} 12010-016-2206-5$

Ramos, A. C. D., Rolemberg, M. P., Moura, L. G. M., Zilio, E. L., Santos, M. D. F., and González, G. (2013). "Determination of solubility parameters of oils and prediction of oil compatibility," Journal of Petroleum Science and Engineering 102, 36-40. DOI: 10.1016/j.petrol.2013.01.008

Si, T. Y. (2012). "Basic principles and applications of solubility parameters (extract)," Cultural Relics Restoration and Research (00), 242-251.

You, C. J., and Shi, X. H. (2001). "Predict miscibility of polymer blends by the solubility parameter," Polymer Materials Science and Engineering 2001(1), 162-165. DOI: 10.3321/j.issn:1000-7555.2001.01.042

Zhang, Q., Tan, X., Wang, W., Yu, Q., Wang, Q. Miao, C., Guo, Y., Zhuang, X., and Yuan, Z. (2019). "Screening solvents based on Hansen solubility parameter theory to depolymerize lignocellulosic biomass efficiently under low temperature," ACS Sustainable Chemistry \& Engineering 7(9), 8678-8686. DOI: 10.1021/acssuschemeng.9b00494

Zhao, L., Wang, Q., and Ma, K. (2019). "Solubility parameter of ionic liquids: A comparative study of inverse gas chromatography and Hansen solubility sphere," ACS Sustainable Chemistry \& Engineering 7(12), 10544-10551. DOI: 10.1021/acssuschemeng.9b01093

Article submitted: April 28, 2021; Peer review completed: July 11, 2021; Revised version received and accepted: September 3, 2021; Published: September 9, 2021.

DOI: $10.15376 /$ biores.16.4.7112-7121 